\title{
GPDs for non zero skewness in transverse and longitudinal position space
}

\author{
Harleen Dahiya ${ }^{* \dagger}$ \\ Department of Physics, Dr. B.R. Ambedkar National Institute of Technology, Jalandhar, 144011, \\ India \\ E-mail: dahiyah@nitj.ac.in
}

\section{Narinder Kumar}

Department of Physics, Dr. B.R. Ambedkar National Institute of Technology, Jalandhar, 144011, India

\begin{abstract}
We investigate the the spin non-flip $H(x, \zeta, t)$ and spin flip $E(x, \zeta, t)$ part of GPDs for the particle conserving $n \rightarrow n$ overlap in the DGLAP region $(\zeta<x<1)$ by expressing them in terms of overlaps of light front wave functions (LFWFs). The Fourier transform (FT) of the GPDs w.r.t. to the transverse momentum transfer as well the FT of the GPDs w.r.t. $\zeta$ have also been obtained giving the distribution of partons in the transverse position space and the distribution in the longitudinal position space respectively.
\end{abstract}

XV International Conference on Hadron Spectroscopy-Hadron 2013

4-8 November 2013

Nara, Japan

* Speaker.

${ }^{\dagger}$ Authors would like to thank S.J. Brodsky and O.V. Teryeav for helpful discussions. 


\section{Introduction}

We intercept the Deep Virtual Compton scattering (DVCS) $\gamma^{*}(q)+p(P) \rightarrow \gamma\left(q^{\prime}\right)+p\left(P^{\prime}\right)$ at the partonic level through the concept of Generalized Parton Distributions (GPDs) [1]. The GPDs are experimentally accessed through the overlap of DVCS and Beith-Heitler process. Several experiments, such as, H1 collaboration [1, 2, 3], ZEUS collaboration [4, 5] and fixed target experiments at HERMES [6] have finished taking data on DVCS. Experiments are also being done at JLAB, Hall A and B [7] and COMPASS at CERN [8] to access GPDs. GPDs not only allow us to access partonic configurations with a given longitudinal momentum fraction but also at specific (transverse) location inside the hadron. GPDs depend on three variables $x, \zeta$, , where $x$ is the fraction of momentum transferred, $\zeta$ gives the longitudinal momentum transfer and $t$ is the square of the momentum transfer in the process. GPDs are much richer in content and carry much more information about the hadron structure than ordinary parton distributions.

GPDs can be expressed as overlaps of light-front wavefunctions (LFWFs) of the target hadron in light front gauge [9]. Polynomiality is satisfied if one considers the LCWFs of simple spin 1/2 objects like dressed quark or a dressed electron in theory. We have generalized the framework of QED by assigning a mass $M$ to external electrons in the Compton scattering process, but a different mass $m$ to the internal electron line and a mass $\lambda$ to the internal photon line [10]. In the present work, we have calculated the $H$ and $E$ for non zero skewness. To understand the significance of the momentum fraction carried by the quarks in the process, we have considered the cases for fixed $x$ and different values of $\zeta$ as well as for fixed $\zeta$ and different values of $x$. Both GPDs $H$ and $E$ are studied in longitudinal and transverse position space by taking Fourier transform w.r.t. $\zeta$ and $\Delta_{\perp}$ giving the distribution of partons in the longitudinal position space and the distribution in the transverse position space respectively.

\section{Kinematics of DVCS and Generalized Parton Distributions}

In the DVCS, the frame is specified by choosing a convenient parametrization of the light-cone coordinates for the initial and final proton [9]

$$
P=\left(P^{+}, \overrightarrow{0_{\perp}}, \frac{M^{2}}{P^{+}}\right), \quad P^{\prime}=\left((1-\zeta) P^{+},-\overrightarrow{\Delta_{\perp}}, \frac{M^{2}+\vec{\Delta}_{\perp}^{2}}{(1-\zeta) P^{+}}\right),
$$

where $M$ is the proton mass. The four momentum transfer from the target is $\Delta=P-P^{\prime}=$ $\left(\zeta P^{+}, \overrightarrow{\Delta_{\perp}}, \frac{t+\vec{\Delta}_{\perp}^{2}}{\zeta P^{+}}\right)$, where $t=\Delta^{2}$. In addition, overall energy-momentum conservation requires $\Delta^{-}=P^{-}-P^{\prime-}$, which connects $\vec{\Delta}_{\perp}^{2}, \zeta$ and $t$ as $t=2 P \cdot \Delta=-\frac{\zeta^{2} M^{2}+\vec{\Delta}_{\perp}^{2}}{1-\zeta}$. The generalized form factors $H, E$ are defined through the matrix elements of the bilinear vector currents [9]

$$
\begin{aligned}
& \left.\int \frac{d y^{-}}{8 \pi} e^{l x P^{+} y^{-} / 2}\left\langle P^{\prime}\left|\bar{\psi}(0) \gamma^{+} \psi(y)\right| P\right\rangle\right|_{y^{+}=0, y^{\perp}=0} \\
& =\frac{1}{2 P^{+}} \bar{U}\left(P^{\prime}\right)\left[H(x, \zeta, t) \gamma^{+}+E(x, \zeta, t) \frac{l}{2 M} \sigma^{+\alpha}\left(-\Delta_{\alpha}\right)\right] U(P) .
\end{aligned}
$$

The off-forward matrix elements can be expressed as overlaps of the light front wave functions [11]. For non-zero skewness $\zeta$ there are diagonal parton number conserving contributions in the 
kinematical region $\zeta<x<1$ and $\zeta-1<x<0$ and there are parton number changing contributions in the region $0<x<\zeta$. If we consider a spin $1 / 2$ target state consisting of a spin 1 particle and a spin $1 / 2$ particle, the contribution to the spin flip conserving and non conserving part of GPDs in the domain $\zeta<x<1$ can be expressed as

$$
\begin{gathered}
\frac{\sqrt{1-\zeta}}{1-\frac{\zeta}{2}} H_{2 \rightarrow 2}(x, \zeta, t)-\frac{\zeta^{2}}{4\left(1-\frac{\zeta}{2}\right) \sqrt{1-\zeta}} E_{2 \rightarrow 2}(x, \zeta, t)= \\
\int \frac{d^{2} \overrightarrow{k_{\perp}}}{16 \pi^{3}}\left[\psi_{+\frac{1}{2}+1}^{\uparrow *}\left(x^{\prime}, \overrightarrow{k_{\perp}^{\prime}}\right) \psi_{+\frac{1}{2}+1}^{\uparrow}\left(x, \overrightarrow{k_{\perp}}\right)+\psi_{+\frac{1}{2}-1}^{\uparrow *}\left(x^{\prime}, \overrightarrow{k_{\perp}^{\prime}}\right) \psi_{+\frac{1}{2}-1}^{\uparrow}\left(x, \overrightarrow{k_{\perp}}\right)+\psi_{-\frac{1}{2}+1}^{\uparrow *}\left(x^{\prime}, \overrightarrow{k_{\perp}^{\prime}}\right) \psi_{-\frac{1}{2}+1}^{\uparrow}\left(x, \overrightarrow{k_{\perp}}\right)\right] \\
\frac{1}{\sqrt{1-\zeta}} \frac{\Delta^{1}-\imath \Delta^{2}}{2 M} E_{2 \rightarrow 2}(x, \zeta, t)= \\
\int \frac{d^{2} \overrightarrow{k_{\perp}}}{16 \pi^{3}}\left[\psi_{+\frac{1}{2}+1}^{\uparrow *}\left(x^{\prime}, \overrightarrow{k_{\perp}^{\prime}}\right) \psi_{+\frac{1}{2}+1}^{\downarrow}\left(x, \overrightarrow{k_{\perp}}\right)+\psi_{+\frac{1}{2}-1}^{\uparrow *}\left(x^{\prime}, \overrightarrow{k_{\perp}^{\prime}}\right) \psi_{+\frac{1}{2}-1}^{\downarrow}\left(x, \overrightarrow{k_{\perp}}\right)\right]
\end{gathered}
$$

where

$$
x^{\prime}=\frac{x-\zeta}{1-\zeta}, \overrightarrow{k_{\perp}^{\prime}}=\overrightarrow{k_{\perp}}-\frac{1-x}{1-\zeta} \overrightarrow{\Delta_{\perp}}
$$

We calculate the GPDs in simulated model of hadron LFWFs with the two-particle wave function for spin up and spin down electron expressed in Ref. [11]. Differentiating the QED LFWFs with respect to $M^{2}$ improves the convergence of the wave functions at the end points of $x$ as well as the $k_{\perp}^{2}$ behavior. We differentiate $\varphi\left(x, \overrightarrow{k_{\perp}}\right)$ with respect to $M^{2}$ and have

$$
\varphi^{\prime}\left(x, \overrightarrow{k_{\perp}}\right)=\frac{e}{\sqrt{1-x}} \frac{1}{\left(M^{2}-\frac{{\overrightarrow{k_{\perp}}}^{2}+m^{2}}{x}-\frac{{\overrightarrow{k_{\perp}}}^{2}+\lambda^{2}}{1-x}\right)^{2}} .
$$

The helicity non-flip GPD $H$ as well as the helicity flip term $E$ can be calculated in this model.

\section{GPDs in position space}

The FT with respect to the transverse momentum transfer $\Delta_{\perp}$ gives the GPDs in transverse impact parameter space. We introduce $b_{\perp}$ conjugate to $\Delta_{\perp}$ giving

$$
\begin{aligned}
\mathscr{H}\left(x, \zeta, b_{\perp}\right) & =\frac{1}{(2 \pi)^{2}} \int d^{2} \Delta_{\perp} e^{-l \Delta_{\perp} \cdot b_{\perp}} H(x, \zeta, t) \\
& =\frac{1}{2 \pi} \int \Delta d \Delta J_{0}(\Delta b) H(x, \zeta, t), \\
\mathscr{E}\left(x, \zeta, b_{\perp}\right) & =\frac{1}{(2 \pi)^{2}} \int d^{2} \Delta_{\perp} e^{-l \Delta_{\perp} \cdot b_{\perp}} E(x, \zeta, t) \\
& =\frac{1}{2 \pi} \int \Delta d \Delta J_{0}(\Delta b) E(x, \zeta, t),
\end{aligned}
$$

where $\Delta=\left|\Delta_{\perp}\right|$ and $b=\left|b_{\perp}\right|$. 
Similar to the transverse momentum transfer, following Ref. [12], we introduce a longitudinal boost invariant impact parameter $\sigma$, which is conjugate to longitudinal momentum transfer $\zeta$. A boost invariant impact parameter conjugate to the longitudinal momentum transfer is defined as $\sigma=\frac{1}{2} b^{-} P^{+}$. The GPDs in longitudinal position space are now expressed as

$$
\begin{aligned}
& \mathscr{H}(x, \sigma, t)=\frac{1}{2 \pi} \int_{0}^{\zeta_{\max }} d \zeta e^{\imath \frac{1}{2} P^{+} \zeta b^{-}} H(x, \zeta, t), \\
& \mathscr{E}(x, \sigma, t)=\frac{1}{2 \pi} \int_{0}^{\zeta_{\max }} d \zeta e^{l \frac{1}{2} P^{+} \zeta b^{-}} E(x, \zeta, t) .
\end{aligned}
$$

In the region $\zeta<x<1$, the upper limit of integration is given by $\zeta_{\max }$

$$
\zeta_{\max }=\frac{-t}{2 M^{2}}\left(\sqrt{1+\frac{4 M^{2}}{(-t)}}-1\right),
$$

whereas in the region $0<x<\zeta$, the upper limit is given by the value of $x$.

Diffraction pattern is obtained for both $\mathscr{H}(x, \sigma, t)$ and $\mathscr{E}(x, \sigma, t)$ where $\zeta_{\max }$ plays the role of the slit width. Since the position of the minima (measured from the center of the diffraction pattern) is inversely proportional to the slit width, the minima move away from the center as the slit width $\left(\zeta_{\max }\right)$ decreases. In both the plots the primary maxima is followed by secondary maxima and as the value of $|t|$ increases, the minima moves towards smaller value of $\sigma$, which is a characteristic of diffraction pattern obtained in single slit experiment.

\section{Conclusions}

In the present work we have studied the GPDs in transverse and longitudinal position space. We have used a two body model which represents a composite system consisting of a fermion and vector boson with arbitrary masses. The calculations have been done with non-zero $\zeta$ in the DGLAP region $x>\zeta$. We have considered the $n \rightarrow n$ parton number conserving overlap. Differentiating the denominator of the light front wave function w.r.t $M^{2}$ improves the convergence near the end points of $x$. The impact parameter space representations are obtained by taking the Fourier transform of the GPDs with respect to transverse momentum transfer. Further, if we introduce a longitudinal boost invariant impact parameter $\sigma$ conjugate to longitudinal momentum transfer $\zeta$, after Fourier transform, we get the GPDs in longitudinal position space. Both $H$ and $E$ show the diffraction pattern analogous to diffractive scattering of a wave in optics where the distribution in $\sigma$ space. However, in order to get the full Lorentz invariant picture in longitudinal space, one has to study the GPDs in $x<\zeta$ region as well.

\section{References}

[1] For reviews on generalized parton distributions, and DVCS, see M. Diehl, Phys. Rept, 388, 41 (2003); X.Ji. Phys. G 24 , 1181 (1998); A.V. Radyushkin, hep-ph/0101225, K. Goeke, M.V. Polyakov, M. Vanderhaeghen, Prog. Part. Nucl. Phys. 47, 401 (2001).

[2] C. Adlloff et al. (H1 Collaboration), Eur. Phys. J. C 13, 371 (2000). 
[3] C. Adlloff et al. (H1 Collaboration), Phys. Lett. B 517, 47 (2001).

[4] J. Breitweg et al.(ZEUS Collaboration), Eur. Phys. J. C 6, 603 (1999).

[5] S. Chekanov et al. (ZEUS Collaboration), Phys. Lett. B 573, 46 (2003).

[6] A. Airpetian et al. (HERMES Collaboration), Phys. Rev. Lett. 87, 182001 (2001).

[7] S. Stepanyan et al. (CLAS Collaboration), Phys. Rev. Lett. 87, 182002 (2001).

[8] N. D’Hose, E. Burtin, P.A.M. Guichon, J. Marroncle (COMPASS Collaboration), Eur. Phys. J. A 19, 47 (2004).

[9] S.J. Brodsky, M. Diehl, D.S. Hwang, Nucl. Phys. B 596, 99 (2001).

[10] S.J. Brodsky and S.D. Drell, Phys. Rev. D 22, 2236 (1980).

[11] S.J. Brodsky, D.S. Hwang, B. Ma, I. Schmidt, Nucl. Phys. B 593, 311 (2001).

[12] S.J. Brodsky, D. Chakrabarti, A. Harindranath, A. Mukherjee, J.P. Vary, Phys. Lett. B 641, 440 (2006); Phys. Rev. D 75, 0143003 (2007). 